\title{
L'ÉTYMOLOGIE PORTUGAISE SELON JOHN MINSHEU (1617) ${ }^{1}$
}

Dans le cadre de nos travaux sur l'évolution du lexique portugais (v. "Dictionnaire chronologique portugais" 1976; "História do léxico português" 1990) nous avons commencé non seulement à analyser les périodiques portugais entre 1700 et 1850 (v. Messner/Aglassinger 1992) sinon aussi à réunir des oeuvres lexicographiques anciennes. Nous espérons pouvoir publier un jour un "dictionnaire des dictionnaires portugais" (c'est pour l'espagnol qu'existe une telle oeuvre, restée malheureusement fragmentaire: le "Tesoro lexicográfico" de Samuel Gili Gaya, Madrid 1947-1952). Dans les "Beihefte" de Lusorama (Messner 1993), nous publierons un échantillon qui embrasse l'espace de temps limité par la publication du dictionnaire de Rafael Bluteau en 1712 ("Vocabulario Portuguez et Latino, etc., autorizado com exemplos dos melhores escritores portuguezes, e latinos, e offerecido a El Rey de Portugal, D. João V pelo Padre D. Rafael Bluteau, Coimbra 1712") et la 6e édition du dictionnaire de Antonio de Moraes Silva en 1858 (Diccionario da Lingua Portugueza composto por A. de Moraes Silva, Lisboa 1858.).

Les premiers dictionnaires portugais dont l'auteur est Jerónimo Cardoso datent du milieu du $16 \mathrm{e}$ siècle. Cardoso a été caractérisé comme "Celui qui fut l'Antonio de Nebrija du Portugal" (Teyssier 1990). C'est en 1611 qu'apparaît un "Dictionarium lusitanico-latinum" (Agostinho Barbosa), et en 1634, Bento Pereira publie "Prosodia in Vocabularium Trilingue, Latinum, Lusitanum et Castellanum", suivi, en 1647, du "Thesouro da lingua portugueza". Ces travaux de Pereira sont rapidement devenus les oeuvres de base et ont connu plusieurs éditions (la lère est de 1750). C'est enfin en 1712, qu'apparaît le dictionnaire de Bluteau qui allait devenir le point de référence de tous les lexicographes postérieurs.

A côté de ces dictionnaires existent aussi d'autres oeuvres qui fournissent des renseignements très précieux pour la connaissance du lexique portugais; il est curieux de constater que ces livres n'ont pas encore été analysés quant aux premières datations de beaucoup de mots portugais. A notre connaissance, personne n'a pas encore utilisé le "Vocabulario que se sigue en seys lenguas, de los nombres de los Arboles, yervas, frutas, y otras cosas contenidas en el presente Libro de los secretos de Agricultura" que Frei Miguel Agustín avait ajouté à son oeuvre "Libro de los secretos de la agricultura casa de campo y pastoril". Ce livre, d'abord écrit en catalan, avait été traduit en espagnol en 1626.

$1 \quad$ Nous remercions le Fonds zur Förderung der Wissenschaftlichen Forschung, Wien, pour le support qu'il nous donne. 
Dans un livre qui décrit l'histoire de la lexicographie catalane, les auteurs ne peuvent pas donner d'information précise d'où venaient les connaissances lexicologiques de Augustín: "No resulta fàcil d'esbrinar on degué informar-se Agustí per compondre aquestes llistes" (Colón/Soberanas 1985, 102). Ils ne pouvaient pas le faire parce qu'un autre livre ne leur avait pas été assez bien connu: il s'agit de la traduction espagnole d'un livre pharmaceutique de l'antiquité. Ce texte espagnol apparaissait pour la première fois en 1555 sous le titre:“ Pedacio Dioscorides Anazarbeo, Acerca de la materia medicinal...traduzida de lengua Griega, en la vulgar Castellana...por el Doctor Andrés de Laguna."

Sur plusieurs centaines de pages sont décrites des plantes et des animaux (v. les réproductions ci-jointes). Pour le lexique portugais, il est important de constater que presque tous les petits chapitres monographiques contiennent une rubrique appellée: Nombres. Et là, on trouve souvent le terme en plusieurs langues: en grec, en latin classique, en latin vulgaire ou tardif, en arabe, en espagnol, en catalan, en italien, en français, en allemand et en portugais. Nous avons réuni tous les mots portugais de Laguna (à peu près la moitié des mots espagnols est accompagnée d'un mot portugais), et nous publions cette liste en ordre alphabétique dans le "Beiheft" de Lusorama, ci-devant cité.

Ce vocabulaire nous permet de constater que Miguel Agustín avait copié Laguna. Là où Laguna n'avait pas donné le mot portugais correspondant au mot espagnol, Agustín aussi ne le mentionnait pas non plus. Exemple: Laguna: Lat. Rapum \& Rapa, Ar. Seliem, Cast. Nabo redondo, Cat. Naps redons, It. Rape, Fr. Rave, Tud. Ruben. Agustín: Lat. Rapum, Cast. Nabo redondo, Cat. Naps redons, It. Rape, Fr. Rave.

D'autre part, Laguna permet de rétrodater toute une série de mots portugais enregistrés dans les dictionnaires historiques et étymologiques modernes (Machado 1977; Cunha 1982): valeriana, amieiro, oregão, etc. (les trois mots sont datés pour 1813 dans Cunha 1982).

Nous n'avons pas non plus rencontré des références à une autre oeuvre, un dictionnaire volumineux de plus de 500 pages: John Minsheu publiait à Londres, en 1617, un dictionnaire sous le titre "Ductor in Linguas, The Guide into Tongues", où il fait suivre les mots anglais des formes correspondantes en dix langues, dont le portugais: il paraît que ce soit le repertoire le plus riche en étymologies portugaises au $17 \mathrm{e}$ siècle. Ce livre permet, d'ailleurs, de donner à beaucoup de mots portugais une nouvelle datation.

C'est surtout des expressions scientifiques qui apparaissent attestées pour la première fois dans Minsheu: corrosivo (1813), coscinomancia (XVIII), etc. (les dates sont celles de Cunba 1982).

La source portugaise de Minsheu n'est pas encore connue: il cite souvent les oeuvres du fameux lexicographe Calepin, mais celui-ci ne mentionne pas la langue portugaise dans ses dictionnaires multilingues. Un exemple de Minsheu 66b est le suivant: 
CHALKE. Br(itish) Cálch. G(allicum = French) Croye, ou Craye, f. I(talian) Créta, Créda . H(ispanicum = Spanish) Grédo, Cal. f. à Lat: Calx, cis, idem, q. calida à calore, of the heat of it.

$\mathrm{T}$ (eutonick) kreide. $\mathrm{B}$ (elgick) kryd. L(atine) Créta, ae vel à Creta insula, in qua optima provenit: vel à crescendo, quoniam proprietas ejus est, ut macerando crescat. $\S$ Calep.: of the Iland of Crete, where the best Chalke groweth. G(reeke)...

Les dictionnaires de Cardoso n'ont pas non plus été utilisés par Minsheu. Dans le titre complet, notre auteur dit: "Ductor in linguas cum illarum barmonia et etimologiis, originationibus, rationibus et derivationibus in omnibus his undecim linguis."

Il n'est pas vrai que tous les mots portugais sont accompagnés de leur étymologie immédiate, mais au 17e siècle, le lecteur instruit de l'oeuvre de Minsheu ne pouvait pas manquer d'établir une relation entre le mot portugais et son homologue latin: la forme portugaise p.ex. contraer est accompagnée de la latine contrahere, et le mot portugais bragas du latin braccae, etc.

Plus important pour 1'historie de la philologie portugaise est le fait que Minsheu donne toute une série d'explications étymologiques explicites d'un certain nombre de mots. Nous reproduisons dans la liste suivante des 227 mots que nous avons rencontrés sur les 100 premières pages tous ceux qui commencent avec la lettre $A$ (34). Cela permettra de suivre les progrès de la recherche étymologique, par ex. en comparant les explications de Minsheu avec celles d'autres chercheurs. Nous citons de temps en temps Cunha 1982, Machado 1977, et en contraste, Constancio 1836.

acrecentar 23a: ab accrescendo (Cunha 1982: do latim " accrescentare baseado em accrescens)

açujentar 93a: à çujo, i. sordidus (Constancio: assujentar de sujar).

acustumarse 5a: ex ad \& consuetudinem (Constancio: a \& costumar; Machado 1977: de acostume).

àdro 72a: à Lat: ager (Constancio: Lat. atrium; Cunha 1982: do lat. atrium).

afagar 69b: fortè à gr. agalliao, i. exulto, laetitia gestio (Constancio: do grego eu, bem, e kharis, amor, carinho; Cunha 1982: do arabe).

albernóz 64a: Vox maura (Constancio: ar.; Cunha 1982: ar.).

alcachofre 35a: vox Arabica (Constancio: ar.; Cunha 1982: do ár. hisp.).

alcoviteiro 33b: Vox Arabica (Constancio: de alcoveto; Machado 1977: de alcovitar). algália 74b: vox Barbara (Constancio: ar.; Cunha 1982: ar.).

algodam 57a: dict: quod ex illa arbore proveniat quam Barbari Cotum vocant. (Constancio: ar.; Cunha 1982: ár. hisp.).

almofaça 84b: ab Arab: Almohaza. (Constancio: em Cast. almorzar, do Alem. morgen, manham e essen, comer; Cunha 1982: ár.).

almoxarife 31a: vox arabica (Constancio: ar.; Cunha 1982: ár.). 
alsine 70a: Gr. alsine, dicta est quod in lucis nascatur quos Graeci alse vocant. (Machado 1977: lat.-gr.).

alvayade 66a: ab Arab. Bayad (Constancio: ar.; Cunha 1982: ár.).

alvo 58b: à lat. albo signo quod in scopo ponitur (Constancio: lat.; Cunha 1982: lat.). ameas 33b: Vox arabica (Constancio: do lat. moenia; Cunba 1982: mina + a).

amizade 5a: ab amicitia (Constancio: do Cast.; Cunha 1982: lat. ${ }^{*}$ amicitatem). amorinho 80b: dim. ab amore.

amorteçer 39a: à morte (Constancio: a pref., morte, e ecer des.; Cunha 1982: de morte).

ampola 55b: à Lat: ampulla (Constancio: lat.; Cunha 1982: lat.).

apertar $97 \mathrm{~b}$ (recte $79 \mathrm{~b}$ ): ab apertus (Constancio: a pref., perto, ar des. inf.; Cunha 1982: do lat. appectorare).

arca 19a: ab arcere (Constancio: lat. arca; Cunha 1982: lat. arca.).

arribar 20a: ex arriba, i. supra (Constancio: a pref., riba do lat. ripa, praia, ar des. inf.;

Cunha 1982: lat. arripare).

artilleria 20a: fortè ab arte quia est ars terribilis, aut á Gallico quasi Art tirerie, i. tirer par art (Constancio: do fr.; Cunha 1982: franc.).

assalto 21a: ab assiliendo (Constancio: lat.; Machado 1977: ital.).

assossego 60a: ab assossegar, i. tranquillare, sedare (Constancio: v. socegar).

atadura 41b: ab atar, i. ligar (Constancio: de atar).

ataúd 81a: fortè à Lat: Tumba, aut ab Hisp: ataviar, to make fit, quia accomodatur cadaveri (Constancio: ar.; Cunha 1982: ár.).

ataviar 7a: a voce Lat. aptare (Constancio: ar.; Cunha 1982: gótico).

atavio 50a: ab aptare (Constancio: de ataviar; Cunha 1982: der. regr. de ataviar).

atirar 64a: à Lat: attrahere (Constancio: a pref., tiro, ar des. inf.; Cunha 1982: origem desconhecido).

atrevimento 23a: ex atrever, i. audere (Constancio: do verbo).

avisado 73b: à Lat. visus (Constancio: do verbo: Cunha 1982: do verbo).

avisar 7b: à Gal. adviser (Constancio: do franc. aviser; Cunha 1982: do fr.).

azemel 62b: ab agenda, i. conducenda mula (Constancio: ar.; Cunha 1982: ár.).

Notre spécimen de 34 mots contient, selon Minsheu, 14 mots d'origine latine, 9 d'origine arabe et 6 dérivés. La correspondance entre Minsheu et les autres étymologistes est très grande pour les mots arabes et français, tandis que Minsheu énumère beaucoup plus de mots d'origine latine que Constancio ou Machado. Constancio est, d'ailleurs, le seul qui explique des mots portugais par une provenance espagnole (l'exemple le plus curieux est almorço). Il faut dire, que là où l'origine d'un mot n'était pas si claire, Minsheu construisait une étymologie assez artificielle. 
Nous espérons avoir pu, par ces quelques lignes, montrer l'importance d'analyser les anciens textes lexicographiques du portugais.

\title{
Bibliographie:
}

G. Colón/A. J. Soberanas, Panorama de la lexicografía catalana, Barcelona: Encicl.Catalana, 1985.

Fr. Solana Constancio, Novo Diccionario critico e etymologico da Lingua Portugueza, Paris: A.F. Carneiro, 1836.

A.G. Guncha, Dicionário etimológico da Língua Portuguesa, Rio: Nova Fronteira, 1982.

S. Gili Gaya, Tesoro lexicográfico, Madrid: CSIC, 1947-1952.

J.P. Machado, Dicionário Etimologico da Língua Portuguesa, Lisboa: Confluência, 1977.

D. Messner, Dictionnaire chronologique portugais, Heidelberg: Winter, 1976.

D. Messner, História do léxico português, Heidelberg: Winter, 1990.

D. Messner, Ein “dicionário dos dicionários portugueses" in: Beihefte zu Lusorama, 1993.

D. Messner/J. Aglassinger, Die portugiesische Sprache zwischen 1700 und 1850, ein Forschungsprojekt, in: Lusorama 17/1992, 14-25.

J. Minsheu, Ductor in Linguas, The Guide into Tongues etc., Londini: J. Browne, 1617 (Scholar's Fascimilies \& Reprints, Delmar New York, 1978).

P. Teyssier, Jerónimo Cardoso et les origines de la lexicographie portugaise, in: id., Etudes de Littérature et de Linguistique, Paris: Fund. Gulbenkian, 1990, 199230.

\author{
Povzetek \\ PORTUGALSKE ETIMOLOGIJE JOHNA MINSHEUA (1617)
}

\footnotetext{
Avtor ugotavlja, da so iberoromanski jeziki v slovarjih šestnajstega, zlasti pa sedemnajstega stoletja bogato zastopani, in obžaluje, da gradivo, ki ga nudijo, ni bilo dovolj izrabljeno. Veliko specializiranih besednjakov, npr. farmakologije, nudi obilico izrazov za rastline in živali, ki sicer v literarnih portugalskih delih, pa tudi kastiljskih in katalonskih, niso izpričani.

Podrobneje je obravnavan desetjezični slovar Johna Minsheua (London, 1617). Dodan je kratek del tega slovarja (besede, ki se začenjajo z glasom $a$-). Nekajkrat je mogoče ugotoviti, da so poznejši etimološki slovarji celo manj zanesljivi.
} 


\section{Illuftrado por el Doet. Lag.}

soxico. De mas defto, fu eftomago rellenado de culantro, y dexado fecar, fi fe beue molido, fo corre ̀̀ las mordeduras de las ferpientes, y es vtil contra la gota coral. La ceniza de toda la Comadreja, quemada en vna olla de ticrra, fi con vinagre fe apliç, es conueniente à la gota. Con fu fangre fe vntan vilimente los lamparones: 12 qual tambien lirue 2 la gota corat

M VSTE L A.

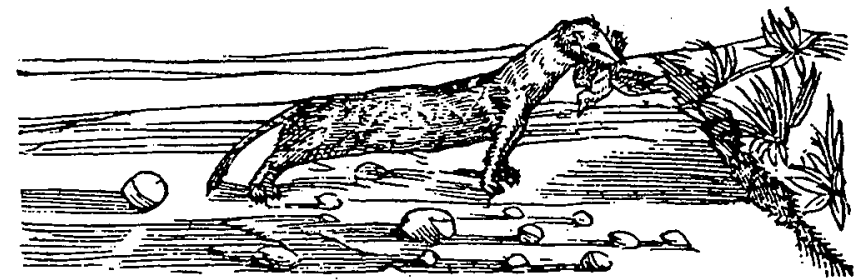

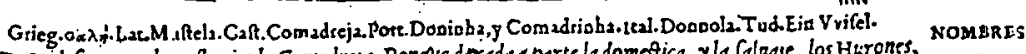

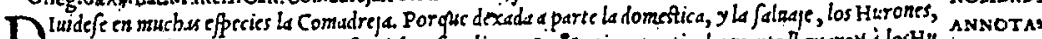

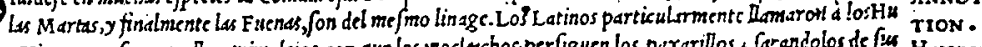
rones viuerras, y for aquelios animalejes, son que los noclatchos perfiguen los puxarillos s farandolos de fur Huroves.

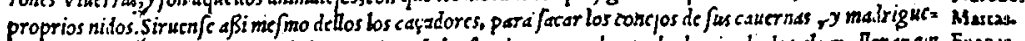

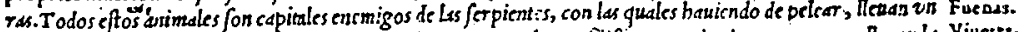

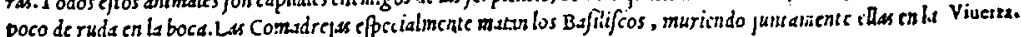
batalla,por razquide fu bitionidez pistif cra.

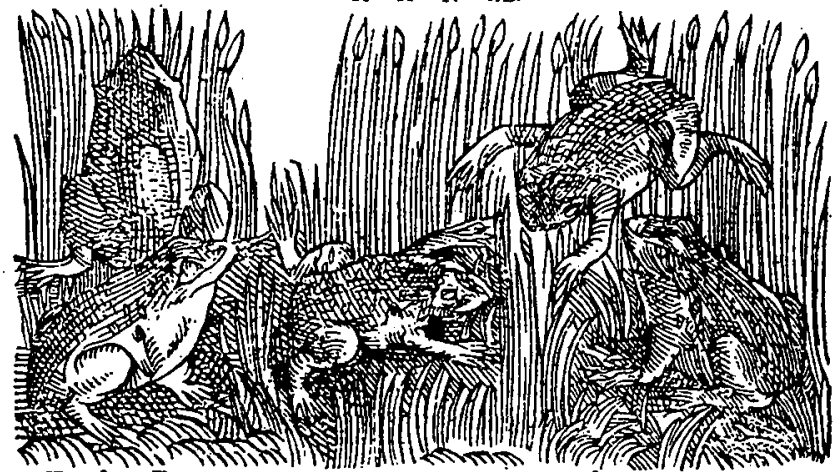

\section{Delas Ranas.}

Cap.XXV.

$T$ As Ranas fritas con azeyre y fal, y comidas anfi con fu caldo, fon remedio fatudable con . Litra qualqquicra ferpiente:y firuen conira los apoltemas de las cucrdas ya inuetcrados. La reniza de les quales poluoreada, reitzana las effufiones de fangre, y aplicada con pez liquiea en forma de vngiaento, haze renacer los cabellos bolados del alopecia. La fangre de las verdes ranas impide gue no renazcan en las cejas loepelos, fi fe inftia cu el proprio lugar del qual fe as. rancaron. El cozimicnto delas heruidus er agaa y vingore : trahido en la boce, mitiga el dolor de los dientes.

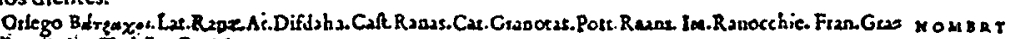
moilley Ralae. Tnd. Eun. Finkeh.

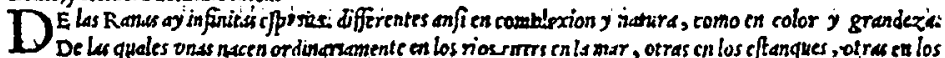
ANmots

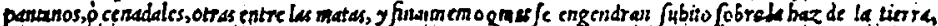

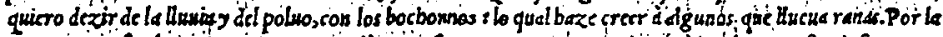

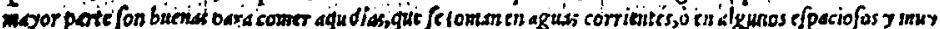




\section{8 \\ Lib.IIII.de Diofc.}

MB RCVRIALIS MAS. MERCVRIALIS POBM
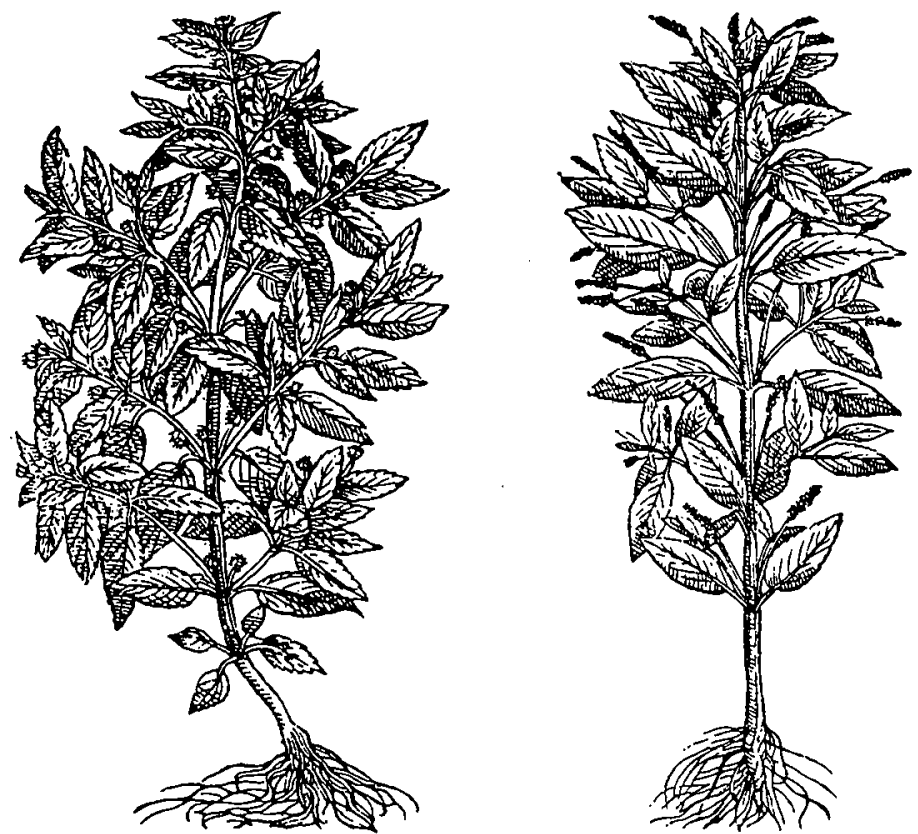

y comida, relaxa el vientre. Cuezenfe en agua, y defpues dafe a beucr lu caldo, para purgar $i_{2}$ colera, y los humores aquoros. Las hojas de la hembra, regun parece, majadas, beuidas, y apli cadas a la natura de la muger, defpues de fu natural purgation, hazen que fe conciba hembra: $y$ las del macho adminiftradas en la melma manera, fon caula que fe engendre varon.

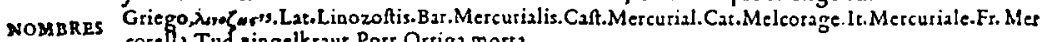
corell.Tud.aingelkraut. Porr. Ortig 2 mort2.

ANNOTA = A Mercurial fe lamo anfi,por hauer fido ballada del Dios Mercurio,y de $\beta$ pues communicada a los bó TION. Lbres. Diuidefe en macho y en hembra. Produzc el macho los granos de fu fimiente dos a dos bermanados por todos los internodlos del allo, quiero dezir of idos en aquel antgulo, que bazen el tallo y el ramillo iz del procede. Mu el fructo de la hembra cömusamëte fe vec falir por Lus extremidades de cada tallo, mamenu do que aquel del macho, empero copiofo, y en forma de raximillos. Las hojas de la bembra fon algo mas blan quezinas quelas del macho: y las rayzes de la ena,y de la otra efpecic muy delgadas, y inutiles. Llamafe cada vma de aqueftas plantas por Caftilla en numero plural Mercuriales:y no ay höbre rico ni pobre g per fon Caincense no las conozcasporque fon muy or dinorias y famlliares en el $v$ fo de los clyfteres: fino preguntenlo a Clara farmo fa cly ftelera de Salemancaila qual folia fiempre en mi tiempo tener tres o quatro tinajas lenas de caldo de azelgas y Mercuriales, aguzado con fal y orines:del qual a tar a cada vna cr bawa cada diddic ftramente cient mil arudacicon que en xug aua los infelices vtentres de aquellos pupilos enfortunados, que ja

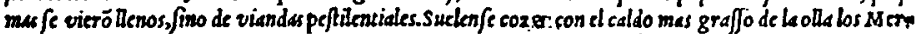
curiales:y defpuesdar fe a beuer vna bora antes del paflo,el lubrico bedrio de allos:el gual rclaxa admirable mëte les restrinidos, a las vezes besta el folo a moucr, y purgar los gibe iad R robarbaro y Ef camonea, no

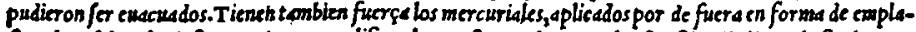
fro, de refoluer las infiammationes,y molificar los apoftemat duros. Del resto, filo sñadido a la fin de aque

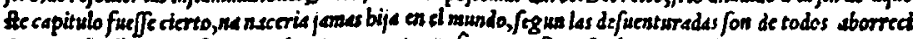

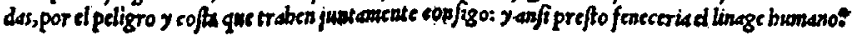

